\title{
Secondary Emotions Deduction from Context
}

\author{
Kuderna-Iulian Bența \\ Communication Department \\ Technical University of Cluj-Napoca \\ Cluj-Napoca, Romania \\ Iulian.Benta@com.utcluj.ro \\ Marcel Cremene \\ Communication Department \\ Technical University of Cluj-Napoca \\ Cluj-Napoca, Romania \\ Marcel.Cremene@com.utcluj.ro
}

\author{
Nicoleta Ramona Gibă \\ Psychology, Personal Development, Social Sciences \\ Babeş-Bolyai University \\ Cluj-Napoca, Romania \\ ramona_giba@yahoo.com \\ Ulises Xolocotzin Eligio \\ Learning Sciences Research Institute \\ University of Nottingham \\ Nottingham, United Kingdom \\ lpxux@nottingham.ac.uk
}

\author{
Anca Rarău \\ Computer Science Department \\ Technical University of Cluj-Napoca \\ Cluj-Napoca, Romania \\ Anca.Rarau@cs.utcluj.ro
}

\begin{abstract}
Human centred services are increasingly common in the market of mobile devices. However, affective aware services are still scarce. In turn, the recognition of secondary emotions in mobility conditions is critical to develop affective aware mobile applications. The emerging field of Affective Computing offers a few solutions to this problem. We propose a method to deduce user's secondary emotions based on context and personal profile. In a realistic environment, we defined a set of emotions common to a museum visit. Then we developed a context aware museum guide mobile application. To deduce affective states, we first used a method based on the user profile solely. Enhancement of this method with machine learning substantially improved the recognition of affective states. Implications for future work are discussed.
\end{abstract}

Keywords- affective computing, context awareness, neural networks, basic and secondary emotions.

\section{INTRODUCTION}

There is a theoretical distinction between basic emotions ${ }^{1}$ and secondary emotions. In [1], Plutchik proposed that in order to survive, human species developed eight basic (or primary) affective states: acceptance, joy, anger, anticipation, disgust, sorrow, fear and surprise. In this account, combinations of these basic emotions generate 28 secondary emotions, e.g. optimism $=$ anticipation + joy. There is no general agreement in regards of what emotions are basic or secondary. However, the basic-secondary distinction is widely accepted in emotion research.

${ }^{1}$ The terms emotion and affective state are used interchangeably throughout this paper.
The field of affective computing has traditionally focused in the recognition of basic affective states (happiness, anger, surprise, fear, sadness, disgust and neutral). In comparison, the recognition of secondary emotions has received less attention. Some advances have been achieved in laboratory settings, e.g. accurate recognition of sadness and amusement from natural expression and personalization over time [2]. But in ever-day life, the contextual information is critical in the definition of secondary emotions e.g. love [3], shame and guilt [4]. As such, the relation between context and secondary emotions is a fairly new interest in the field of affective computing and context awareness mobile technology.

Some researchers claim that the secondary states are exclusively human [5] and culture-dependent [6].

A potential utility of mobile context aware technology is the support of learning in out of school contexts. It allows for control, ownership, learning and continuity between contexts, fun and communication [7]. This utility can be augmented if mobile context aware technology were capable of detecting secondary affective states. It is known that socially intelligent technology can effectively support learning [8] and theoretical studies have shown correlations between affective states and learning outcomes [9]. An affective aware tutoring system that capitalizes on these aspects [10] deals with a set of affective states that are, most of them, secondary (five axis, like anxietyconfidence, frustration-euphoria, with six states each). Moreover, preliminary work [11] focuses on a narrower set of secondary affective states relevant to learning: frustration, confusion and boredom. 
This work presents a method to detect secondary emotions from a mobile device in a typical context of informal learning: a museum. We depicted a scenario where the recognition of secondary emotions enhances the adaptability of a context aware mobile device to the user's personal needs:

Jane is a sensitive person. As she walks around the art gallery she likes listening to music assorted to her affective state. A specially designed device is detecting her state (like calm, enthusiasm, interest) but finally she agrees or not with the detected value. The system will request through her smartphone's mobile connection the song that is most appropriate to her current state, from a long list of affective labelled items stored on a server."

The paper is organized as follows: in the next section we present the related work in secondary affective states detection. Section 3 presents our approach to the relationship between secondary emotions and context. The museum experience we set up as realistic contextual framework is described first. Then we explain the methodology used to define the emotions relevant to this environment and its associated contextual factors. The development of the museum guide test bed is presented in Section 4. In Section 5 we present the procedure and results of two secondary emotion deduction methods. The first is based in a user profile. The second method enhances the first one with a neural network. Future work and conclusions are given in the Section 6 and 7, respectively.

\section{RELATED WORK}

Works that define the relationship between secondary and basic emotions are scarce [12][13][14] and/or based on experiments with a very few people (five in [12]). Secondary states are expressed in [12] as a weighted linear combination of the basic states. The weight values are determined by giving values between -100 and 100 for each. In recent work [13], it has been proposed a fuzzy logic based method to build a unified personalized 3D affective model that allows the representation of the secondary states in relation with the basic ones. They claim that knowing the percentage of each basic affective state in the current emotion (detectable with the actual devices) one may classify it as one of the secondary states based on a personal affective model.

The basic emotions have been the main focus for the researchers in affective computing. But there are a few studies on secondary emotion detection, usually related to a learning context. For example, in [14] the authors proposed the use of a leap chair with pressure sensors in an attempt to discover the student engagement level (high and low interest and take a break). They found nine postures that are relevant in detecting engagement and used HMM to train the system. They reported $87.6 \%$ recognition with data not included in the training set. In another work, [15] a multiple modalities method (facial, postural and from the current activity (gamming)) is proposed for detecting interest (high and low interest, refreshing, bored, neutral and "other"). An average accuracy of $67.8 \%$ is obtained by training a HMM with the values obtained from each separate emotion channel.
Frustration or the need for help is detected from multiple sources using specialised devices like a pressure mouse (frustration), skin conductance sensor (arousal), a posture chair motivation), facial-expression camera (head nod or shake, mouth fidgets, smiles, blink events, and pupil dilations) [16].

\section{THE CORRELATION BETWEEN EMOTIONS AND CONTEXT}

It is common sense that people uses contextual information to figure out which is somebody's current affective state. There are works showing correlations between environmental factors (context) and emotions [17]. But none, as far as we know, has tried to deduce affective states from context using technology.

\section{A. The Contextual Framework: A Museum Experience}

In order to have a realistic and yet easy recordable environment we have chosen first year students in IT\&C as a target group to visit an art museum like room organized in the University. Given the situation of exploring art in a gallery, we decided to determine what would be the list of the most important states felt by the visitors, the list of factors that induce those states and the measure each factor would have to induce a certain emotion

\section{B. Data Collection and Analyses}

1) Empirical Observations of the Affective States and Contextual Factors in the Museum Experience

We observed peoples' affective reactions directly and in video recordings (following facial expressions, movements, body postures and gestures as clues) and we had focus group discussions on feelings when engaging in an art experience in different museums.

Finally we had two outputs: a list of affective states and a list of contextual factors relevant to these affective states. First we defined a list of 22 emotions that appeared the most frequently. We started by using 47 POMS [18] states and then we shrank it to a reasonable list of 22 emotions based on interviewing the ten museum visitors.

The list of emotional states was: Agitated, Amused, Discontented, Curious, Sad, Calm, Aggressive, Depressed, Irritated, Frustrated, Hazy, Alone, Worried, Frightened, Furious, Happy, Indifferent, Relaxed, Interested, Bored, Enthusiastic and Stressed. Secondly we defined a list of 18 binary contextual factors.

2) Predominant Affective States in the Museum Experience A questionnaire with the list of 22 emotions plus three blank spaces was designed. We had left three free spaces for other states to be filled in. In correspondence with each state a set of intensity should have been mentioned: very low intensity, low intensity, medium intensity, high intensity, and very high intensity. The tested group consisted in group of 31 persons, 16 of them were females and their average age was 21.7 years. Most of them were students with an IT\&C background. All of them were Romanian speakers.

Given the fact that we used a number of persons (31) that is statistically relevant for a well defined target group, we think 
that the following results can be generalized in respect to the group's characteristics: age (19-25), background (IT\&C), gender (balanced), students.

The results indicated that only a few states are felt with more than medium intensity, so we calculated a value for each state taking into account the number of occurrences in the 'intense' and 'very intense cases', with a doubled value for the very intense ones, for example, for the state "Relaxed", 10 persons rated this emotion as 'intense' and 5 persons rates it as 'very intense' and the final rate was $10 * 1+5 * 2=20$. The most rated states were: Curious, Calm, Happy, Relaxed, Interested and Enthusiastic, as depicted in the Table 1.

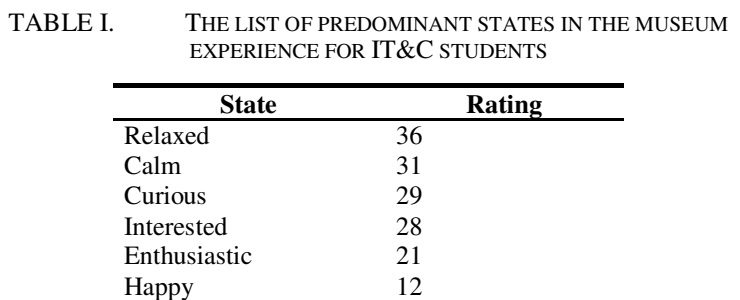

The values for "Curious" and "Interested" are almost the same as the two words meanings are very similar. Among the six states we notice there is just "Happy" that is a basic emotion, the others being secondary. Although we have chosen to include happiness as it had a good rating (12) compared to loneliness (the next item in the rating list), which had only 5.

\section{3) Contextual Factors Influencing Emotions in the Museum Experience}

We set up a questionnaire to relate the aforementioned set of six affective states (table 1) with the set of 18 binary factors determined from empirical observations as explained above. The tested group was different from the one used to define the list of affective states. They were 31 students, 16 of them females, with an average age of 20.23 years and an IT\&C background. The participants were asked to relate each factor to each of the emotions by indicating the extent to what a factor would influence the intensity of a given emotion.

The correlations between factors and context are parameters that change according to target group, kind of museum, person, country but the method of deducing the secondary emotions from context is the same. The data obtained was complex and full explanation is beyond the purpose of this paper. So we focus on two global analyses.

In the first analysis we ranked each factor to notice its participation in inducing intense states. Table 2 shows the results. If positive, the sign in front of the ranking indicates a tendency to induce intense states. If negative, it indicates a tendency to reduce intense states. There are some factors that are ranked near to zero, but they increase the intensity in some states while decreasing the intensity of others.
RANKING FOR FACTORS THAT INDUCE INTENSE EMOTIONS IN AN ART EXPERIENCE.

\begin{tabular}{lll}
\hline No. & \multicolumn{1}{c}{ Factor } & Ranking \\
\hline 1 & The novelty of the paintings & +10 \\
2 & The colors in the paintings & +12 \\
3 & The shapes in the paintings & +9 \\
4 & The novelty of the author/authors & -10 \\
5 & The celebrity of one or more paintings & +60 \\
6 & The celebrity of one or more authors & +37 \\
7 & A short time for the visit & -5 \\
8 & An appropriate duration of time for the visit & +45 \\
9 & A long time for the visit & +44 \\
10 & A small number of visitors in the same room & +20 \\
11 & A medium number of visitors in the same room & +6 \\
12 & A big number of visitors in the same room & 0 \\
13 & The presence of familiar persons & +33 \\
14 & The presence of unknown persons & +1 \\
15 & A sunny day & +19 \\
16 & A rainy day & -14 \\
17 & A cloudy day & -23 \\
18 & A snowy day & +13 \\
\hline
\end{tabular}

In the second analysis, we calculated the probability of each emotion intensity to increase as a function of each listed factor according to the following formula, where $i$ is the index for the intensity level ( $\mathrm{n}=5$ levels), $j$ is the index for the factor $(\mathrm{m}=18$ factors) and $k$ is the index for the affective state ( $\mathrm{p}=6$ states):

$$
\Pi_{\kappa}=\frac{\sum_{i, j=1}^{n, m} \operatorname{rank}_{i j}}{\sum_{i, j, k=1}^{n, m, p} \operatorname{rank}_{i j k}} .
$$

Table 3 shows the results. There seem to be three emotions that predominate among the 18 factors:

- Interested - average intensity 4 (high intensity) in 8 factors from 18

- Curious - average intensity 4 (high intensity) in 5 factors from 18

- $\quad$ Relaxed - average intensity 3.5 (between medium and high intensity) in 4 factors from 18.

TABLE III. THE PROBABILITY FOR EACH STATE TO OCCUR IN THE ART EXPLORING EXPERIENCE AS A FUNCTION OF THE CONTEXTUAL FACTORS

\begin{tabular}{ll}
\hline \multicolumn{1}{r}{ State } & Probability $\mathbf{* 1 0 0 \%}$ \\
\hline Relaxed & 15.596 \\
Calm & 8.869 \\
Curious & 25.075 \\
Interested & 31.804 \\
Enthusiastic & 1.009 \\
Happy & 7.645
\end{tabular}

It is interesting to notice that some states like "Happy" and "Curious" are "negatively" influenced (their intensity is diminished from the reference 'medium intensity' value). In this case the intensity is getting to the value 'low', influenced 
by the novelty of the author, little time to spend and the rainy weather.

The most important impact on the emotions is noticed in the case of celebrity of the panting and the celebrity of the author/s. The time available has "positive" effects on "Curious", "Interest" and "Calm" inducing high intensity, but has a "negative" effect on "Enthusiasm", "Happy" and "Curious" when it is not enough.

The bad weather ${ }^{2}$ (rainy, cloudy) impact negatively the "Relax" and "Calm", rated as "low intensity" by many participants. A big number of factors have decreased the values for the intensity of "Relax" and "Calm". We may notice a positive correlation between "Calm" and "Relax" all over the factors.

"Curious" and "Interest" seem to be induced by the novelty of the paintings, the shapes and the celebrity of the authors and paintings and are rated as "high intensity" by the most participants.

\section{The Museum Guide Test BeD}

The work is based on some previous research done in context aware museum guides [19][20]. Our application has two parts: one is a web based application for profiling the user and the other one is the context aware mobile guide. In the following we explain the functionality of each part.

\section{A. The User Profile}

We have designed and implemented a web based application for gathering personal data from the users. It is a simple web application that displays static pages for the questionnaire, dynamically generates pages with questions related to the museum content and it stores the results in a MySQL relational database. The server side is written in PHP.

At first the user has to register and then s/he can have password protected access to the private area. Then the user fills up a questionnaire with 18 questions, one for each 18 contextual factors, allowing the user to indicate the correlation between each factor and the six emotions and for each state, five levels of intensity. Finally another page is generated with questions about: a) the list of known artists in the current gallery; b) the list of painting titles; c) the type of colour preference (warm or cold); d)the preferred shape types (dead or alive nature) where the participant has to make a choice.

\section{B. The Context Aware Mobile Guide}

The idea was to have a location aware like museum guide for a test bed in order to be able to monitor the values of the context (number of persons in the room, weather, current painting details etc.) and to predict the current predominant state.

The indoor localisation is still a research issue, even if there are already some good solutions [19][21]. The use of an indoor tracking solution for smart phones was not mandatory for our

\footnotetext{
2 The experiments took place in Romania in winter and spring 2008.
}

experiment. However we have had recordings on what was the current painting as the user should indicate the next artwork by pressing direction buttons in order the server to send the appropriate content.

The other context elements monitored were:

- the number of persons located in the same room

- the relations between those persons

- weather information

- the duration

The experimenter would have to set the values for the weather, relations between the persons and the maximum duration of time for the experiment on a web based GUI

The information about the number of persons simultaneously present in the system was deduced from the number of users currently logged in.

Basically we developed a simple client-server application. On the client side, we had a J2ME application and we used two smart phones (Nokia 6630 and N95) to deploy it. The user entered the name and password and then navigated the content as walking around the room. The pictures where included in the deployment file, but a network connection was needed for the text content to be downloaded locally and displayed and to send the declared affective state back to the server. We used the wireless LAN connection on N95 and the 3G data connection on the Nokia 6630.

On the server side we had a Java application and a very simple communication protocol with the mobile application. For each current painting the mobile application requested to the server a corresponding content. The server accessed the database and sent it. On the other hand a declared affective state was sent to the server and stored in the Event table in the database, in addition to current context elements and user id.

\section{DEDUCTION OF SECONDARY AFFECTIVE STATES}

We proposed two methods for the deduction of the current affective state: the first one is based on the user profile, extracted from the questionnaire (that the user had to fill before using the guide). The second method consists of improving the first one with an expert system. It learns to predict de affective state taking into account the current contextual factors and the users preferences existing in the profile.

\section{A. The Profile-based Inference method}

In order to predict the current affective state, we extracted the current contextual factors as a vector $\mathrm{F}$ with 18 binary values (for instance given the number of persons is 3 then the value for the $10^{\text {th }}$ factor is 1 , and for the $11^{\text {th }}$ and $12^{\text {th }}$ is 0 ). The users profile extracted from the questionnaire is a three dimensional matrix (factor, affective state, intensity of the affective state) named $\mathrm{P}$. We multiply the $\mathrm{F}$ and $\mathrm{P}$ matrix and obtain a two dimensional matrix (affective states and intensity). The predicted state would be the one that is the most intense (by adding all the intensity values for one affective state). 


\section{1) Results of the Profile-based inference method}

We recorded 231 events (current contextual factors and the declared state and its intensity) for 8 persons in different test scenarios: single experience, small group (2-5 persons), big group. In some case we simulated time pressure (by reducing the time left for the visit). The weather has been different in the three day when we ran the tests, even if we did not cover all possible situations. The exposed paintings where different as well: some of them were famous because of the content (i.e. "The birth of Venus") or of the author (i.e. Paul Cezanne), but usually both (i.e. Leonardo da Vinci, "Mona Lisa"). Variations were in terms of content (landscapes, people) or colours (warm and cold), too.

Using the profile extracted from the questionnaire we obtained 40 correct predictions from 231 events, that is $17.316 \%$

In order to have a reference to compare this result with, we calculated the probability to guess the current affective state: given the fact that the user is in one state (let's say Happy), the probability of a correct guess is $1 / 6$. The probability for a user to have as current states one of the six states is also $1 / 6$. So the probability of guessing the correct current state, not knowing the current states is $1 / 6 * 1 / 6=1 / 32$. That is $3.125 \%$.

Comparing the percentage of correct prediction based on the user profile $(17.316 \%)$ with the percentage of a correct guess $(3.125 \%)$ we may say it is significant, but not satisfactory.

\section{B. The Neural Network-based Inference}

We propose to use an expert system in order to increase the prediction rate for the current affective state. We decided to use a neural network, which turned out to be a good choice as you may see in the results section. The first attempt was to use a simple perceptron with 18 factors +30 values extracted from the profile as input and the six affective states as output. We tested the configuration in Matlab. The results were not satisfactory because the big number of inputs. So we designed and tested a multilayer perceptron. Following is a part of the code with the optimum values for the parameters:

\section{$\% \%$ create MLP}

Ninp $=48$

Nhid $=8$;

inp_domains = repmat $([0$ 9], Ninp, 1);

net $=$ newff(inp_domains, [Nhid Noutp], $\{$ 'tansig' 'purelin' $\}$ );

net.trainParam.epochs $=150$;

$\% \%$ train net

net $=$ train(net, inputs, outputs);

The number of inputs was $18+30=48$, the number of hidden layers was 8 (Nhid) and we used newff function for the feed forward multilayer perceptron with sigmoid and linear step functions. The number of optimal epochs was 150 .

We trained the network with a set of 200 recordings obtained from 8 users. Each recording was a pair of values (input, desired output). As input we had a vector with the actual values for the 18 binary factors and a set of 30 values obtained from the matrix product $\mathrm{F}$ and $\mathrm{P}$. The desired output was the declared state (one of the six).

\section{1) Results of the Neural Network Method}

We have trained the designed neural network, described in Section IV.C.2, with 200 of the 231 recording and we tested it with the remaining 31 values for the percentage of correct prediction.

The results indicate 11 correct predictions out of 31 , that is $35.484 \%$. Even if this result is not as good as expected, it is a good improvement compared to the one obtained with the profile based solution.

We also investigated other classification methods. A comparison of the values obtained for the experimental data ${ }^{3}$ is represented in Fig. 1.

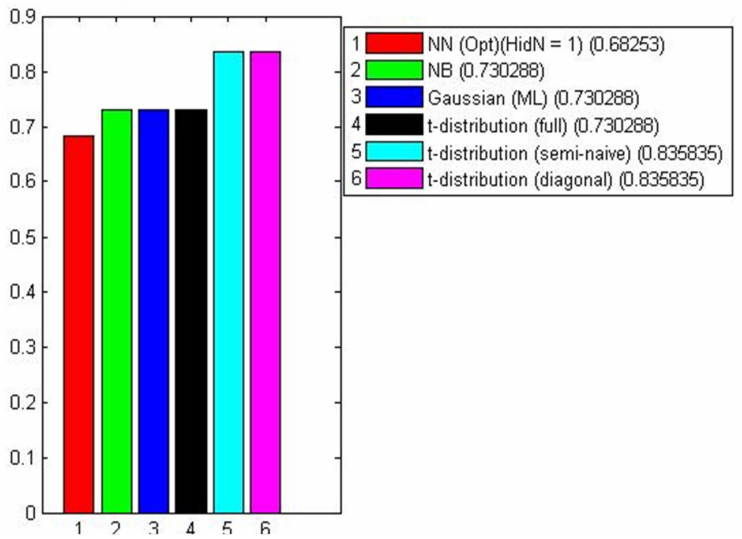

Figure 1. Comparison between different machine learning techniques for the experimental data (the error rate is represented on the y axis)

The input was 200 recordings, 48 input values and 6 classes for the output. The Naïve Based Classifier have had 0.730288 classification rate, the Gaussian Classifier 0.730288 and for the $t$-dist there were three situations:

a) full error rate $=0.730288$

b) semi-naïve error rate $=0.835835$

c) naive error rate $=0.835835$

The optimal value was obtained by the Neural Network Classifier with 8 hidden nodes in one hidden layer. The value obtained is even better then we previously found, that is 0.68253 error rate.

We also performed a $\mathrm{SVM}^{4}$ test on the data with the following parameters:

kernel='gaussian';

kerneloption $=1$;

$\mathrm{C}=10000000$;

verbose $=1$;

lambda $=1 \mathrm{e}-7$;

nbclass $=6$;

The results indicate a $15.50 \%$ of correct classification rate That can be explained as our inputs are in a large number (48).

\footnotetext{
${ }^{3}$ We used a software tool from http://www.patternrecognition.co.za

${ }^{4}$ We used a software from http://asi.insa-rouen.fr/enseignants/ arakotom/toolbox/index.html
} 


\section{FUTURE WORK}

There are two main directions for future work: one concerns the specification of the secondary emotion recognition model. The other implies the machine learning technique.

In designing the model we plan to extend the user profile with some new parameters like the users' personality type and to use them as input for the neural network.

We think the machine learning could be improved if the user would be able to give feedback regarding the system's prediction correctness in deducing his/her affective state. We plan to develop our application to allow online machine learning based on neural networks and user feedback, for instance by using the GARIC [22] neural fuzzy reinforcement system.

\section{CONCLUSIONS}

In this paper we reported the results of a method for detecting secondary affective states from contextual information and personal profile in relation to a museum experience. On the basis of systematic observation in a realistic environment and self-reports, we defined a set of affective states relevant to the museum experience and defined the contextual factors associated with these states. We used these data to model how the contextual factors contribute to increase or decrease the intensity of people's affective states in a museum. Our results in this regard confirm those obtained in previous research on affect, where it has been found that a person's affective states are sensitive to the characteristics of the context.

Moreover, this approach to model the relation between secondary affective states and context allowed us to deduce the user's current affective state. We proposed two methods: the first one based on user profile solely. The second method improved the first one with a machine learning technique. We obtained $17.3 \%$ correct recognition rate with the profile based method and $35.5 \%$ using feed-forward multilayer perceptron with one hidden layer as machine learning technique.

We look forward to increase the deduction power of our method by adding new features in the user profile and by using the user's feedback for online machine learning.

\section{ACKNOWLEDGMENT}

We thank our students (A. Fatiol, O.Litan, R.M. Cimpean, E. Ciurea, M. Herculea) for their help in developing the web based and the J2ME application and to all participants in the tests.

This work benefit by the support of the national contract PN2 Idei number 1062 and CNCSIS type A number 1566.

\section{REFERENCES}

[1] R.A. Plutchik, "A General Psychoevolutionary Theory of Emotions. In: Kellerman", R.P.H. (ed.): Emotion: Theory research and experience, Vol. 1, 1980, pp. 3-33.
[2] J. Bailenson, E. Pontikakis, I. Mauss, J. Gross, M. Jabon, C. Huthcerson, Nass and O. John, "Real-time classification of evoked emotions using facial feature tracking and physiological responses", International Journal of Human-Computer Studies, 66(5), 2008, pp.303-317.

[3] J.B. Nezlek, K. Vansteelandt, I. Van Mechelen, P. and Kupens, "Appraisal-Emotion relationships in daily life", Emotion, 8(1), 2008, pp 145-150.

[4] E.M.W. Tong, G.D. Bishop, H.C. Enkelmann, P.Y. Why and M.S Diong, "Emotion and appraisal: A study using ecological momentary assessment", Cognition and Emotion, vol. 21(7), 2007, pp.1361 - 1381.

[5] S. Demoulin, J.-P. Leyens, M.-P. Paladino, R. Rodriguez-Torres, R.-P. Armando and J.F. Dovidio, "Dimensions of "uniquely" and "nonuniquely" human emotions", Cognition and Emotion, 18(1), 2004, pp. 71-96.

[6] R. Harré, "An Outline of the Social Constructionist Viewpoint", Harre, R. (ed.), The Social Construction of Emotions. Blackwell Publishing, Oxford and New York, 1989, pp. 2-13.

[7] K. Issroff, E. Scanlon, and A. Jones, "Affect and mobile technologies: case studies", Proc. The CSCL alpine rendez-vous, workshop 2: Beyond mobile learning, Trinity College, 2007.

[8] N. Wang, L. Johnson, R. Mayer, P. Rizzon, E. Shaw and H. Collins, "The politness effect: Pedagogical agents and learning outcomes", International Journal of Human-Computer Studies, vol. 66(2), 2008, pp. $98-112$

[9] B. Kort, R. Reilly and R. Picard, "An Affective Model of Interplay between Emotions and Learning: Reengineering Educational PedagogyBuilding a Learning Companion", Proc. Second IEEE International Conference on Advanced Learning Technologies (ICALT'01), 2001.

[10] A. Kapoor, S.Mota and R.W. Picard, "Towards a learning companion that recognizes affect", In Proc. AAAI Fall Symposium, 2001.

[11] S.K. D'Mello, S.D. Craig, B. Gholson, S.Franklin, R. Picard and A. Graesser, "Integrating affect sensors in an intelligent tutoring system", Proc. Affective Interactions: The Computer in the Affective Loop Workshop at 2005 International conference on Intelligent User Interfaces, AMC press, New York, 2005.

[12] T. Yanaru, "An emotion processing system based on fuzzy inference and subjective observations", Proc. 2nd New Zealand Two-Stream International Conference on Artificial Neural Networks and Expert Systems (ANNES '95), Dunedin, New Zealand,1995, pp. 15-21.

[13] K.-I. Bența, H.-I. Lisei and M. Cremene, "Towards a Unified 3D Affective Model", Proc. Consortium Proceedings of International Conference on Affective Computing and Intelligent Interaction (ACII2007), Lisbon, Portugal, 2007.

[14] S. Mota and R.W. Picard, "Automated posture analysis for detecting learner's interest level”, Proc. CVPR Workshop on HCI, 2003.

[15] A. Kapoor, S. Mota and R.W. Picard, "Towards a learning companion that recognizes affect", Proc. AAAI Fall Symposium, 2001.

[16] W. Burleson, "Affective learning companions: strategies for empathetic agents with real-time multimodal affective sensing to foster metacognitive and meta-affective approaches to learning, motivation, and perseverance", PhD. thesis, Massachusetts Institute of Technology, 2006

[17] R. Plutchik, The Psychology and Biology of Emotion. Harper Collins New York, 1994.

[18] D.M. McNair, M. Lorr, L.F. Droppleman, "POMS : Profile Of Mood States", Educational and Industrial Testing Service, 1971.

[19] P. Lonsdale, R. Beale and W. Byrne, "Using context awareness to enhance visitor engagement in a gallery space", McEwan, T., Gulliksen, J.andBenyon, D. (eds.), Proc. People and computers xix - the bigger picture. Proceedings of HCI 2005, Springer, London, 2005, pp. 101-112.

[20] O. Stock, M. Zancanaro, P. Busetta, C. Callaway, A. Krüger, M. Kruppa, T. Kuflik, E. Not and C. Rocchi, "Adaptive, intelligent presentation of information for the museum visitor in PEACH", User Modeling and User-Adapted Interaction vol. 17(3), 2007.

[21] http://www.ekahau.com/

[22] H.R. Berenji, P. Khedkar, "Learning and tuning fuzzy logic controllers through reinforcements", Neural Networks, IEEE Transactions on vol.3, no.5, Sep 1992, pp.724-740. 